\title{
Wave-induced Lagrangian drift in a porous seabed
}

\author{
Jan Erik H. Weber ${ }^{1}$. Peygham Ghaffari ${ }^{2}$
}

Received: 4 June 2021 / Accepted: 4 November 2021

(C) The Author(s) 2021

\begin{abstract}
The mean drift in a porous seabed caused by long surface waves in the overlying fluid is investigated theoretically. We use a Lagrangian formulation for the fluid and the porous bed. For the wave field we assume inviscid flow, and in the seabed, we apply Darcy's law. Throughout the analysis, we assume that the long-wave approximation is valid. Since the pressure gradient is nonlinear in the Lagrangian formulation, the balance of forces in the porous bed now contains nonlinear terms that yield the mean horizontal Stokes drift. In addition, if the waves are spatially damped due to interaction with the underlying bed, there must be a nonlinear balance in the fluid layer between the mean surface gradient and the gradient of the radiation stress. This causes, through continuity of pressure, an additional force in the porous layer. The corresponding drift is larger than the Stokes drift if the depth of the porous bed is more than twice that of the fluid layer. The interaction between the fluid layer and the seabed can also cause the waves to become temporally attenuated. Again, through nonlinearity, this leads to a horizontal Stokes drift in the porous layer, but now damped in time. In the long-wave approximation only the horizontal component of the permeability in the porous medium appears, so our analysis is valid for a medium that has different permeabilities in the horizontal and vertical directions. It is suggested that the drift results may have an application to the transport of microplastics in the porous oceanic seabed.
\end{abstract}

\section{Article Highlights}

- Surface waves in an upper fluid layer induce Lagrangian mean drift in a porous bottom layer.

- For spatially damped waves, the mean Eulerian current in the porous bed may be larger than the Stokes drift.

- Analysis relevant for transport of microplastics in the porous oceanic bottom layer.

Jan Erik H. Weber

j.e.weber@geo.uio.no

1 Department of Geosciences, University of Oslo, Blindern, PO Box 1022, 0315 Oslo, Norway

2 Department of Arctic R\&D, Akvaplan-Niva, Oslo, Norway 
Keywords Lagrangian wave drift · Porous medium flow · Spatial and temporal wave attenuation - Anisotropic porous bottom layer $\cdot$ Transport of microplastics

\section{Introduction}

In a pioneering paper Reid and Kajiura [18] investigate the linear interaction between surface wave motion in a fluid layer of finite depth and the motion in an underlying saturated porous medium of infinite depth. The fluid is inviscid, and the flow in the porous medium is governed by Darcy's law. It is found that the exchange of fluid between the porous layer and the overlying fluid causes the waves to become spatially attenuated.

The present paper takes a new look at this problem, assuming that the porous layer has a finite depth; see also [20]. The main emphasis of the present investigation will be on the mean particle drift in the porous medium. For this purpose, we apply a Lagrangian description of fluid motion in the fluid layer and the porous bed. The novelty here is that the pressure gradient in Darcy's law becomes nonlinear in the Lagrangian description, yielding a direct wave-induced drift in the porous medium. In particular, for spatially damped waves, we consider the effect of radiation stress [12] at the surface on the mean motion. The Lagrangian mean surface gradient must be balanced by the gradient of the radiation stress in this case; see e.g. Phillips [16] for a discussion of this problem in Eulerian terms. Then, the Lagrangian mean drift in the fluid layer is given by the Stokes drift [19]. In the longwave approximation, the horizontal mean pressure gradient is continuous into the porous bed. Accordingly, the gradient of the radiation stress component in long waves induces a mean horizontal drift in the porous layer, in addition to the small Stokes drift.

We also point out that the exchange of mass between the upper fluid and the porous medium may cause temporal attenuation of the waves. This follows directly from the dispersion relation (52) in the "Appendix". Again, through nonlinearity, this leads to a small horizontal Stokes drift in the porous layer, but now damped in time.

The present problem has some interesting parallels to processes in the natural environment. For example, Luthar et al. [13] have found mean currents induced by surface waves in a seagrass meadow. This is of course a more complicated problem, since the fibrous porous matrix is not fixed but is moving in the oscillatory current. A closer similarity to the present problem is the investigation by Webber and Huppert [20, 21], who calculate the wave-induced Stokes drift in corals reefs.

Another important environmental aspect of the present analysis is the application to the transport of microplastics in the porous oceanic bottom layer. Microplastics are defined as plastic fragments less than $5 \mathrm{~mm}$ in length. It originates from surface plastic debris and finally ends up at the ocean bottom. In natural pore systems, like coral reefs, the permeability typically is $K=5 \times 10^{-7} \mathrm{~m}^{2}$ [20,21]. This corresponds to pore dimensions of order centimeters, and we realize that microplastics at the bottom may penetrate the bottom layer due to the oscillatory surface wave field. When inside the porous bottom layer, these plastic fragments may spread horizontally by the wave-induced Lagrangian mean current.

Apart from reefs and beaches, shallow banks in the open ocean are locations where surface waves may induce oscillatory flow at the ocean bottom. For example, on Spitsbergenbanken in the Barents Sea, the minimum water depths are 26-53 $\mathrm{m} \mathrm{[2],} \mathrm{so} \mathrm{the} \mathrm{sea} \mathrm{bottom} \mathrm{is} \mathrm{well} \mathrm{in}$ reach of long ocean swells. Spitsbergenbanken has a porous bottom layer of depth 5-20 m of loose carbonate material consisting of a mixture of shells, stones and gravel [24]. The permeability is typically $K=2.62 \times 10^{-7} \mathrm{~m}^{2}$ [14], corresponding to an average grain diameter of 
about $2 \mathrm{~cm}$. A considerable accumulation of floating debris (mostly plastic) is observed at the nearby Tromsøflaket [3]; see Fig. 1.

This makes Tromsøflaket a potential source of microplastics in the western Barents Sea. The proximity to the shallow Spitsbergenbanken, and the direction of the ocean currents, could lead to the accumulation of microplastics in the porous bottom layer of Spitsbergenbanken. The wave-induced Lagrangian transport would then, depending on the direction of the swell, extend laterally the contaminated region. We discuss this problem in some detail at the end of Sect. 7.

\section{Mathematical formulation}

We study surface gravity waves in a horizontal fluid layer of undisturbed depth $H_{1}$ overlying a fluid-saturated porous layer of thickness $\mathrm{H}_{2}$. The fluid is incompressible and homogeneous in both layers and has density $\rho$. In the fluid, we neglect the effect of friction, while in the porous layer the motion is governed by Darcy's law [1]. We take that the $X$-axis is horizontal and situated at the interface between the porous layer and the fluid layer, while the $Z$-axis is vertical, and positive upwards. The pressure is $P$, and upper and lower layer variables are denoted by subscripts 1 and 2, respectively. The free surface of the upper layer is given by $Z_{1}=H_{1}+\eta(X, t)$. Here we assume that the pressure vanishes, i.e. $P_{1}=0$. The impermeable bottom of the porous layer is given by $Z_{2}=-H_{2}$. This configuration is sketched in Fig. 2 .

We assume that the surface wave in the upper layer has a wavelength $\lambda$ that is much larger than the thickness of both layers, i.e. $\lambda \gg H_{1}, H_{2}$. Then we can assume that the pressure is hydrostatic in the vertical. Hence, in both layers

$$
P_{Z} / \rho=-g
$$

where $g$ is the acceleration due to gravity. Utilizing that the pressure is continuous at the interface between the upper and lower layer, where $Z_{1}=Z_{2}=\xi(X, t)$, we find by integration in the vertical that

Fig. 1 Map of the western Barents Sea. NwAC is the Norwegian Atlantic Current

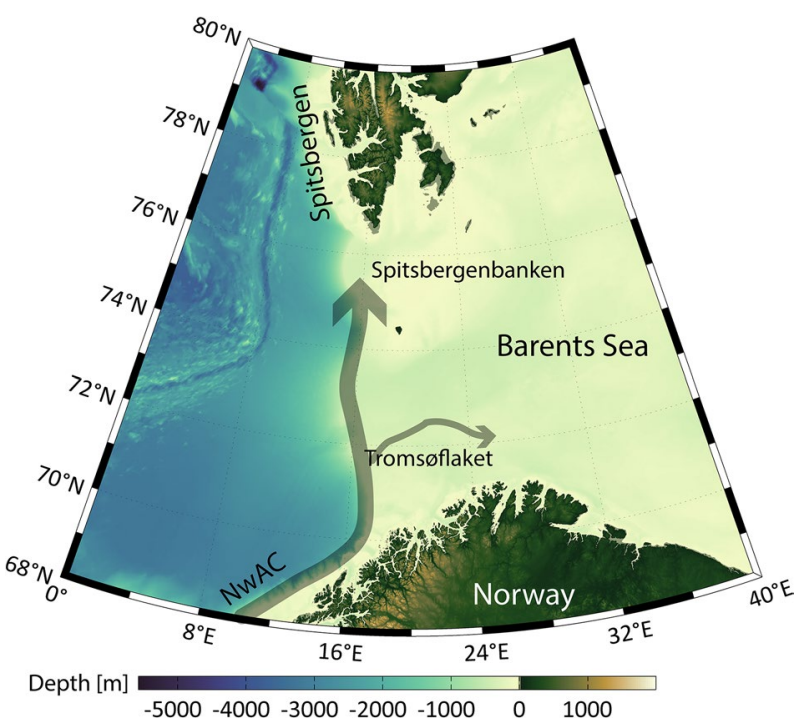

Springer 
Fig. 2 A diagram showing the configuration, with a free surface wave in the upper fluid layer above a porous bed. The floor at $Z_{2}=-H_{2}$ is impermeable



$$
\begin{aligned}
& P_{1} / \rho=-g Z_{1}+g\left(H_{1}+\eta\right), \\
& P_{2} / \rho=-g Z_{2}+g\left(H_{1}+\eta\right) .
\end{aligned}
$$

In the horizontal direction we have for the fluid layer

$$
X_{1 t t}=-g \eta_{X_{1}},
$$

where subscripts denote partial differentiation. In the porous medium, using Darcy's law [1], the momentum equation has the form

$$
\left(v / K_{H}\right) X_{2 t}=-g \eta_{X_{2}},
$$

where $v$ is the kinematic viscosity of the fluid in the pores, and $K_{H}$ is the horizontal component of the permeability of the porous medium. We note right away that the vertical component of the permeability $K_{V}$ does not enter the problem because of the shallow-water assumption.

For the mathematical analysis, we apply Lagrangian coordinates. Let a fluid particle $(a, c)$ initially have coordinates $\left(X_{0}, Z_{0}\right)$. Its position $(X, Z)$ at later times will then be a function of $a, c$ and time $t$. Velocity components and accelerations are given by $\left(X_{t}, Z_{t}\right)$ and $\left(X_{t t}, Z_{t t}\right)$, respectively. It is trivial to express Eqs. (4) and (5) in terms of the independent Lagrangian spatial coordinates $(a, c)$; see [9]. These equations now become

$$
\begin{gathered}
X_{1 t t} X_{1 a}=-g \eta_{a}, \\
\left(v / K_{H}\right) X_{2 t} X_{2 a}=-g \eta_{a} .
\end{gathered}
$$

We note that because of the shallow-water assumption, the horizontal displacements $X_{1}, X_{2}$ are not functions of the Lagrangian independent vertical variable $c$. Furthermore, it is interesting to notice that the governing Eq. (7) in the porous medium becomes nonlinear in the Lagrangian formulation. Even though $(a, c)$ is not the initial particle position, it is convenient to write the displacements of the form (e.g., Pierson [17])

$$
X_{1,2}=a+x_{1,2}(a, t), \quad Z_{1,2}=c+z_{1,2}(a, c, t),
$$

where the deviations $\left(x_{1,2}, z_{1,2}\right)$ can be expanded in series after a small parameter $\varepsilon$ proportional to the wave amplitude [23]. Equations (6) and (7) can thus be written

$$
\begin{gathered}
x_{1 t t}\left(1+x_{1 a}\right)=-g \eta_{a}, \\
\left(v / K_{H}\right) x_{2 t}\left(1+x_{2 a}\right)=-g \eta_{a} .
\end{gathered}
$$


The conservation of mass (here volume) leads to [9],

$$
J(X, Z) \equiv X_{a} Z_{c}-X_{c} Z_{a}=J\left(X_{0}, Z_{0}\right) .
$$

From Eqs. (8), (11) becomes in each layer

$$
x_{t a}+z_{t c}+\left(x_{a} z_{c}\right)_{t}-\left(x_{c} z_{a}\right)_{t}=0 .
$$

In the Lagrangian formulation, the boundary of the upper layer is given by $c=H_{1}$. This is a freely moving material surface, along which the pressure must be constant. At the horizontal bottom boundary of the porous bed, given by $c=-\mathrm{H}_{2}$, the vertical velocity must vanish. Particles originally situated at the horizontal separation line between the fluid layer and the porous bed are given by $c=0$. After the perturbation by the surface wave, these particles will still be found at $c=0$, irrespective of whether they are located in the fluid or in the porous layer. The complicating factor is that the Darcy velocity in the porous medium is defined as an average, which makes the problem of mass and momentum transfer far from trivial; see the comprehensive discussion by Lācis et al. [8]. We here follow the approach in [7, 10], and take that normal component of the velocity and the normal stress (here the pressure) are continuous at the interface, which characterizes a material surface.

Although Eqs. (9) and (10), together with the continuity Eq. (12), are nonlinear, it is seen that the hydrostatic approximation simplifies the problem considerably. In addition, as noted before, only the permeability in the horizontal direction enters the problem. This is an advantage since it appears that the permeability in nature is often anisotropic.

\section{The Lagrangian mean drift}

The linear problem is trivial, and the details have been deferred to the "Appendix". Here we consider the nonlinear problem to second order for the Lagrangian mean quantities. The mean is defined by averaging over the wave cycle, and this process is denoted by an overbar. From Eq. (9), we obtain to second order in the fluid layer

$$
u_{1 t}^{(L)}=-\overline{\tilde{x}_{1 t t} \tilde{x}_{1 a}}-g \bar{\eta}_{a},
$$

where real parts are used for the linear variables and the Lagrangian mean drift in the fluid is defined as $u_{1}^{(L)}=\bar{x}_{1 t}$. Longuet-Higgins [11] derived the Stokes drift $u_{1}^{(S)}$ to second order in Eulerian variables. In Lagrangian terms it can be written

$$
u_{1}^{(S)}=\overline{\tilde{x}_{1} \tilde{x}_{1 t a}}+\overline{\tilde{z}_{1} \tilde{x}_{1 t c}}=-\overline{\tilde{x}_{1 t} \tilde{x}_{1 a}}-\overline{\tilde{z}_{1 t} \tilde{z}_{1 a}},
$$

where we in the last expression have utilized that the flow is irrotational, i.e. $\tilde{x}_{1 c}=\tilde{z}_{1 a}$. It should be pointed out that for irrotational flow, Eames and McIntyre [5] have given an exact expression for the Stokes drift (not limited to second order in wave steepness).

In the Lagrangian formulation the Stokes drift is generally obtained from the governing equations by allowing for a small viscosity (whatever small, as long as it is non-zero). This singular behavior is discussed in the review [23]. In the present inviscid case, the vorticity vanishes in the fluid layer. By expressing this fact in Lagrangian terms, the Stokes drift can be calculated for irrotational waves $[4,22]$.

We define the mean quantity $\bar{Q}$ by 


$$
\bar{Q} \equiv-\overline{\tilde{x}_{1 t t} \tilde{x}_{1 a}}-\overline{\tilde{z}_{1 t t} \tilde{z}_{1 a}} \text {. }
$$

In the shallow-water approximation, the last term on the right-hand side is negligible, and we notice that $\bar{Q}$ appears in Eq. (13). Generally, by simple manipulations

$$
\bar{Q}=-\left(\overline{\tilde{x}_{1 t} \tilde{x}_{1 a}}+\overline{\tilde{z}_{1 t} \tilde{z}_{1 a}}\right)_{t}+\frac{1}{2}\left(\overline{\tilde{x}_{1 t}^{2}}+\overline{\tilde{z}_{1 t}^{2}}\right)_{a} .
$$

We note from Eq. (14) that the first term in Eq. (16) is the time derivative of the Stokes drift. The second term is the spatial gradient of the kinetic energy per unit depth and density for long gravity waves. Since we have equal partition of kinetic and potential energy in nonrotating waves, we can write for the total mechanical energy $E$,

$$
E=\rho H_{1}\left(\overline{\tilde{x}_{1 t}^{2}}+\overline{\tilde{z}_{1 t}^{2}}\right)
$$

Hence, in Eq. (16),

$$
\bar{Q}=u_{1 t}^{(S)}+\frac{1}{2 \rho H_{1}} E_{a}
$$

We finally utilize that wave energy $E$ and wave mean momentum $M$ are connected by

$$
E=C_{0} M=C_{0} \rho U_{1}^{(S)},
$$

(Phillips [16]). Here $U_{1}^{(S)}$ is the vertically integrated Stokes drift (the Stokes flux) in the fluid layer, and $C_{0}=\omega / k=\left(g H_{1}\right)^{1 / 2}$ is the phase speed (and the group velocity) of long waves. Since Eqs. (18) and (19) also are valid in the long-wave approximation, we obtain by insertion into Eq. (13) that

$$
u_{1 t}^{(L)}=-g \bar{\eta}_{a}+u_{1 t}^{(S)}+\frac{1}{2 H_{1}} C_{0} U_{1 a}^{(S)} .
$$

This equation for the Lagrangian drift in high-frequency long gravity waves is also found in [23], (Eq. 114), when the effects of viscosity and the Earth's rotation are disregarded.

In the porous layer, we obtain from Eq. (10) to second order

$$
u_{2}^{(L)}=-\overline{\tilde{x}_{2 t} \tilde{x}_{2 a}}-g K_{H} \bar{\eta}_{a} / \nu,
$$

where $u_{2}^{(L)}=\bar{x}_{2 t}$ is the horizontal Lagrangian mean drift in the porous bed. We observe from Eq. (14) that the first term on the right-hand side of Eq. (21) is just the Stokes drift for long waves in the porous bed, and we can write

$$
u_{2}^{(L)}=u_{2}^{(S)}-g K_{H} \bar{\eta}_{a} / \nu
$$

We thus see that the surface mean gradient in the fluid layer yields an additional drift contribution in the porous bed. It will be demonstrated in Sect. 5 that the surface gradient is proportional to the tensor component of radiation stress per unit density in shallow-water waves when the mean flow is steady. 


\section{Drift in temporally damped waves}

When $\alpha=0$ in Eq. (42), the drift problem becomes particularly simple. We can then assume that the mean variables do not depend on the horizontal coordinate $a$. Hence, from Eq. (20) we find

$$
u_{1}^{(L)}=u_{1}^{(S)}=\frac{1}{2 H_{1}^{2}} C_{0} A^{2} e^{-2 \beta t},
$$

where we have inserted from the "Appendix" for the real part of the variables to obtain $u_{1}^{(S)}$ from Eq. (14). We note that the horizontal Lagrangian mean flow in this case is just the Stokes drift.

In the porous layer Eq. (22) reduces to

$$
u_{2}^{(L)}=u_{2}^{(S)}=\frac{1}{2 H_{1}^{2}} R^{2} C_{0} A^{2} e^{-2 \beta t},
$$

where the small parameter $R$ is defined by Eq. (55). Again, the Lagrangian mean flow in the porous bed equals the Stokes drift. We notice that the Stokes drift here is a factor $R^{2}$ smaller than the Stokes drift in the fluid layer.

We also remark that since $\bar{x}_{t a}=0$ in both layers, we obtain from the continuity Eq. (12) that $\bar{z}_{t c}=0$. With a rigid bottom at $c=-H_{2}$, and continuity of mean velocities at $c=0$, this means that $\bar{z}_{t}=0$ in both layers. In the next section where we consider spatially damped waves, we shall see that this change and we have vertical mean drift velocities in the porous bed as well as in the fluid layer.

\section{Drift in spatially damped waves}

For spatially damped waves, i.e., $\beta=0$ in Eq. (43), we have that $\bar{\eta}_{a} \neq 0$. A steady balance in Eq. (20) then requires

$$
g \bar{\eta}_{a}=\frac{1}{2 H_{1}} C_{0} U_{1 a}^{(S)} .
$$

Following Longuet-Higgins and Stewart [12], we can write the tensor component $S^{(22)}$ of radiation stress per unit density in shallow-water waves along the 1-axis as

$$
S^{(22)}=E /(2 \rho)=C_{0} U_{1}^{(S)} / 2,
$$

where we have used Eq. (19). Hence, from Eq. (25) we find

$$
g \bar{\eta}_{a}=S_{a}^{(22)} / H_{1}
$$

This means that the mean pressure gradient at the surface due to the presence of spatially damped waves must be balanced by the radiation stress gradient per unit depth in order to achieve steady horizontal mean motion. In fact, this balance must hold also in the case when the wave amplitude varies slowly in time.

Utilizing Eq. (25), we find from Eq. (20) in the upper fluid in the long-wave approximation 


$$
u_{1}^{(L)}=u_{1}^{(S)}=\frac{1}{2 H_{1}^{2}} C_{0} A^{2} e^{-2 \alpha a}
$$

Again, the Lagrangian horizontal mean flow in the fluid layer is just the Stokes drift, exactly as it was for temporally damped waves; see Eq. (23).

Now the Stokes flux becomes

$$
U_{1}^{(S)}=\int_{0}^{H_{1}} u_{1}^{(S)} d c=\frac{1}{2 H_{1}} C_{0} A^{2} e^{-2 \alpha a} .
$$

Hence, from Eq. (25) we obtain for the mean surface elevation that

$$
\bar{\eta}=\frac{1}{4 H_{1}} A^{2} e^{-2 \alpha a}
$$

where $A$ in this case is a slowly varying function of time, as will be demonstrated in Sect. 6.

Using the spatial damping rate given by Eq. (56), we can write Eq. (22) in the porous bed, when we insert from Eq. (25),

$$
u_{2}^{(L)}=u_{2}^{(S)}-g K_{H} \bar{\eta}_{a} / v=\frac{1}{4 H_{1}^{2}} C_{0} R^{2}\left[2+H_{2} / H_{1}\right] A^{2} e^{-2 \alpha a} .
$$

We remark that the effect of the radiation stress on the horizontal Lagrangian drift in the porous bed (last term in the brackets) will dominate when $\mathrm{H}_{2}>2 \mathrm{H}_{1}$.

\section{Vertical mean drift for spatially damped waves}

Continuity in the porous layer yields $\bar{x}_{2 t a}=-\bar{z}_{2 t c}$ from Eq. (12). By integrating in the vertical, and using that $\bar{z}_{2 t}\left(c=-H_{2}\right)=0$, we find

$$
w_{2}^{(L)}=\frac{1}{4 H_{1}^{3}} \omega H_{2} R^{3}\left(2+H_{2} / H_{1}\right)\left(c+H_{2}\right) A^{2} e^{-2 \alpha a} .
$$

where $w_{2}^{(L)}=\bar{z}_{2 t}$ is the vertical Lagrangian mean drift in the porous bed. We note that this is very small.

From Eq. (12) we find in the fluid layer to second order

$$
w_{1 c}^{(L)}=\bar{z}_{1 t c}=-u_{1 a}^{(L)} .
$$

Integration in the vertical yields

$$
w_{1}^{(L)}\left(c=H_{1}\right)-w_{1}^{(L)}(c=0)=2 \alpha H_{1} u_{1}^{(L)} .
$$

The kinematic boundary condition is

$$
w_{1}^{(L)}(c=0)=w_{2}^{(L)}(c=0) .
$$

Hence, we have that 


$$
w_{1}^{(L)}\left(c=H_{1}\right)=w_{2}^{(L)}(c=0)+2 \alpha H_{1} u_{1}^{(L)} .
$$

From Eq. (32) we note that the first term on the right-hand side of Eq. (36) is of $O\left(R^{3}\right)$ compared to the second term, which is of $O(R)$ from Eq. (28). Hence, the first term can be neglected. We may then write

$$
w_{1}^{(L)}\left(c=H_{1}\right)=\bar{\eta}_{t}=2 \alpha H_{1} u_{1}^{(L)} .
$$

Inserting from Eqs. (28) and (30) into Eq. (37), we find

$$
\left(A^{2}\right)_{t}-4 \alpha C_{0} A^{2}=0 .
$$

Accordingly, in the case of spatially damped waves the mean free surface has the form

$$
\bar{\eta}=\frac{1}{4 H_{1}} A_{0}^{2} e^{-2 \alpha\left(a-2 C_{0} t\right)} .
$$

Here $A_{0}$ is the initial wave amplitude at $a=0$, where the surface wave enters our domain. We thus see that the free surface in this case has a small upward motion in addition to the small horizontal attenuation.

\section{Discussion and concluding remarks}

It is worth noting that if we require a steady position of the free surface, i.e. $w_{1}^{(L)}\left(c=H_{1}\right)=0$, this would imply that $w_{2}^{(L)}(c=0)=-2 \alpha H_{1} u_{1}^{(L)}$ from Eq. (36). However, by integrating the continuity equation in the porous bed, we find $w_{2}^{(L)}(c=0)=2 \alpha_{2} u_{2}^{(L)}$ which combines to yield

$$
u_{2}^{(L)}=-H_{1} u_{1}^{(L)} / H_{2} .
$$

This means that the volume fluxes for the drift in each layer would be equal in magnitude and oppositely directed. However, this is highly unrealistic. The mean flow in a porous medium must be much smaller than in the fluid above, since the Reynolds number based on the pore diameter must be of order 1 or less for Darcy's law to be valid [1, 15]. In fact, the vertical drift velocity at the interface between the porous bed and the fluid must depend directly on the pore diameter through the permeability; see Eq. (32). When the pore diameter approaches zero, and hence $K_{H} \rightarrow 0$, the interface becomes impermeable, which requires $w_{1}^{(L)}(c=0)=w_{2}^{(L)}(c=0)=0$. This shows that the free surface position cannot be stationary when we consider drift due to spatially damped waves in this problem.

Assuming that marine microplastic particles are non-inertial and passive, our results in Sect. 5 may be applied to Spitsbergenbanken, which has a lateral dimension of about $100 \mathrm{~km}$; see Fig. 1 . We take $H_{1}=30 \mathrm{~m}$ for the ocean layer, and $H_{2}=10 \mathrm{~m}$, $K_{H}=2.62 \times 10^{-7} \mathrm{~m}^{2}$ and $v=10^{-6} \mathrm{~m}^{2} \mathrm{~s}^{-1}$ for the porous bed. Furthermore, we consider a long swell of period $16 \mathrm{~s}$ (wavelength $400 \mathrm{~m}$ ) and typical amplitude $1 \mathrm{~m}$, arriving at the bank from the Norwegian Sea. From (28) and (31), this swell will for a period of $24 \mathrm{~h}$ cause a net horizontal drift of particles above the bed of about $1200 \mathrm{~m}$. In the porous bed, the corresponding drift distance will be about $15 \mathrm{~m}$. From the spatial damping rate (56) such waves will typically attenuate over a distance of $4 \mathrm{~km}$, so swell due to repeated storm 
events in the Norwegian Sea will cause accumulation of microplastics at the western part of the bank.

To the authors' knowledge, the application of a Lagrangian description to flow in porous media is not common. It yields directly the mean particle drift in oscillatory wave motion. Of particular interest is the application of the long-wave approximation, which is relevant for a considerable range of physical and geophysical applications. As pointed out before, a consequence of this approximation is that the vertical component of the permeability of the porous medium does not enter the problem. This simplifies the analysis of the drift in the porous bed considerably. In addition, our results are valid even if the medium is anisotropic.

\section{Appendix}

\section{The linear problem}

We linearize the governing equations and denote the linear variables by a tilde. The surface wave may attenuate in time or in space, and we take that

$$
\tilde{\eta}=A e^{i \kappa a-n t},
$$

where $A$ is the real wave amplitude, and

$$
\begin{aligned}
& \kappa=k+i \alpha, \\
& n=\beta+i \omega .
\end{aligned}
$$

Here $k$ is the real wave number, and $\omega$ the wave frequency, while $\alpha$ and $\beta$ are the spatial and temporal damping coefficients, respectively. We assume slow damping, i.e., $\alpha / k \ll 1$ and $\beta / \omega \ll 1$. From Eq. (9) in the fluid:

$$
\tilde{x}_{1 t t}=-g \tilde{\eta}_{a}
$$

Hence

$$
\tilde{x}_{1}=-\left(i g \kappa / n^{2}\right) \tilde{\eta}
$$

From the continuity Eq. (11):

$$
\tilde{z}_{1 c}=-\tilde{x}_{1 a}=-\left(g \kappa^{2} / n^{2}\right) \tilde{\eta} .
$$

Integrating in the vertical, and using that $\tilde{z}_{1}\left(c=H_{1}\right)=\tilde{\eta}$, we find that

$$
\tilde{z}_{1}=\left[1+g \kappa^{2}\left(H_{1}-c\right) / n^{2}\right] \tilde{\eta} .
$$

In the porous bed:

$$
\tilde{x}_{2 t}=-g K_{H} \tilde{\eta}_{a} / \nu=-\left(i g \kappa K_{H} / \nu\right) \tilde{\eta} .
$$

Hence 


$$
\tilde{x}_{2}=\left[i g \kappa K_{H} /(n v)\right] \tilde{\eta} .
$$

From the continuity equation in the porous bed:

$$
\tilde{z}_{2 c}=-\tilde{x}_{2 a}=\left[g \kappa^{2} K_{H} /(n v)\right] \tilde{\eta} .
$$

Integrating in the vertical, and applying that $z_{2}\left(c=-H_{2}\right)=0$, we find

$$
\tilde{z}_{2}=\left[g \kappa^{2} K_{H}\left(H_{2}+c\right) /(n v)\right] \tilde{\eta} .
$$

Finally, at the interface we must have that $\tilde{z}_{1}(c=0)=\tilde{z}_{2}(c=0)$ (see the discussion at the end of Sect. 2), which yields the complex dispersion relation:

$$
n^{2}-n g \kappa^{2} K_{H} H_{2} / v+g \kappa^{2} H_{1}=0 .
$$

\section{Temporally damped waves}

We first take that $\alpha=0$. Then, to lowest order from the real and imaginary part of Eq. (52), respectively:

$$
\omega^{2}=g H_{1} k^{2},
$$

and

$$
\beta=\frac{\omega H_{2}}{2 H_{1}} R,
$$

where we have defined the small dimensionless parameter $R$ by

$$
R=\omega K_{H} / \nu .
$$

According to Reid and Kajiura [18], $R$ is the fundamental parameter of the porous problem.

\section{Spatially damped waves}

Now we take that $\beta=0$ in Eq. (52). Again, the frequency to lowest order becomes as before; see Eq. (53), while the spatial damping rates becomes

$$
\alpha=\frac{k H_{2}}{2 H_{1}} R .
$$

It is easily seen that for the next approximation we have

$$
\omega^{2}=g H_{1} k^{2}+O\left(R^{2}\right) .
$$

Since long waves are nondispersive, i.e. $C_{g}=C_{0}=\left(g H_{1}\right)^{1 / 2}$, we readily find from Eqs. (54) and (56) for the damping rates that 


$$
\beta=C_{g} \alpha,
$$

as first shown by Gaster [6]. Finally, real values of Eqs. (45), (47), (49) and (51) are used when we calculate the forcing terms for the nonlinear mean solutions.

Acknowledgements Financial support from the Research Council of Norway through the Grant 280625 (Dynamics of floating ice) and Grant 288079 (MALINOR) is gratefully acknowledged.

Funding Open access funding provided by University of Oslo (incl Oslo University Hospital). Not applicable.

Availability of data and material Not applicable.

Code availability Not applicable.

\section{Declarations}

Conflict of interest The authors declare no conflict of interest.

Open Access This article is licensed under a Creative Commons Attribution 4.0 International License, which permits use, sharing, adaptation, distribution and reproduction in any medium or format, as long as you give appropriate credit to the original author(s) and the source, provide a link to the Creative Commons licence, and indicate if changes were made. The images or other third party material in this article are included in the article's Creative Commons licence, unless indicated otherwise in a credit line to the material. If material is not included in the article's Creative Commons licence and your intended use is not permitted by statutory regulation or exceeds the permitted use, you will need to obtain permission directly from the copyright holder. To view a copy of this licence, visit http://creativecommons.org/licenses/by/4.0/.

\section{References}

1. Bear J (1972) Dynamics of fluids in porous media. American Elsevier Publishing Company, New York

2. Belleca VK, Bøe R, Bjarnadóttira LR, Albretsen J, Dolan M, Chand S, Thorsnes T, Jakobsen FW, Nixon C, Plassen L, Jensen H, Baeten N, Olsen H, Elvenes S (2019) Sandbanks, sandwaves and megaripples on Spitsbergenbanken, Barents Sea. Mar Geol 416:105998

3. Buhl-Mortensen L, Buhl-Mortensen P (2017) Marine litter in the Nordic Seas: distribution composition and abundance. Mar Pollut Bull 125:260-270

4. Clamond D (2007) On the Lagrangian description of steady surface gravity waves. J Fluid Mech 589:433-454

5. Eames I, McIntyre ME (1999) On the connection between Stokes drift and Darwin drift. Math Proc Camb Philos Soc 126:171-174

6. Gaster M (1962) A note on the relation between temporally-increasing and spatially-increasing disturbances in hydrodynamic stability. J Fluid Mech 14:222-224

7. Kandel HN, Pascal JP (2013) Inclined film flow with bottom filtration. Phys Rev E 88:052405

8. Lācis U, Sudhakar Y, Pasche S, Bagheri S (2020) Transfer of mass and momentum at rough and porous surfaces. J Fluid Mech 884:A21

9. Lamb H (1932) Hydrodynamics, 6th edn. Cambridge University Press, Cambridge

10. Levy T, Sanchez-Palencia E (1975) On the boundary conditions for fluid flow in porous media. Int J Eng Sci 13:923-940

11. Longuet-Higgins MS (1953) Mass transport in water waves. Philos Trans R Soc Lond A 245:535-581

12. Longuet-Higgins MS, Stewart RW (1960) Changes in the form of short gravity waves on long waves and tidal currents. J Fluid Mech 186:321-336

13. Luthar M, Coutu S, Infantes E, Fox S, Nepf H (2010) Wave-induced velocities inside a model seagrass bed. J Geophys Res 115:C12005

14. Massel SR (2013) Modelling flow in the porous bottom of the Barents Sea shelf. Oceanologia 55:129-146 
15. Palm E, Weber JE, Kvernvold O (1972) On steady convection in a porous medium. J Fluid Mech 54:153-161

16. Phillips OM (1977) The dynamics of the upper ocean, 2nd edn. Cambridge University Press, Cambridge

17. Pierson WJ (1962) Perturbation analysis of the Navier-Stokes equations in Lagrangian form with selected solutions. J Geophys Res 67:3151-3160

18. Reid RO, Kajiura K (1957) On the damping of gravity waves over a permeable sea bed. Trans Am Geophys Union 38:662-666

19. Stokes GG (1847) On the theory of oscillatory waves. Trans Camb Philos Soc 8:441-455

20. Webber JJ, Huppert HE (2020) Stokes drift in coral reefs with depth-varying permeability. Philos Trans R Soc A 378:20190531

21. Webber JJ, Huppert HE (2021) Stokes drift through corals. Env Fluid Mech. https://doi.org/10.1007/ s10652-021-09811-8

22. Weber JE (2011) Do we observe Gerstner waves in wave tank experiments? Wave Motion 48:301-309

23. Weber JE (2019) Lagrangian studies of wave-induced flows in a viscous ocean. Deep-Sea Res Part II 160:68-81

24. Węsławski JM, Kędra M, Przytarski J, Kotwicki L, Ellingsen I, Skardhamar J, Renaud P, Goszczko I (2012) A huge biocatalytic filter in the centre of Barents Sea shelf? Oceanologia 54:325-335

Publisher's Note Springer Nature remains neutral with regard to jurisdictional claims in published maps and institutional affiliations. 